\title{
Present Worth Analysis Using Hesitant Fuzzy Sets
}

\author{
Cengiz Kahraman*, Sezi Çevik Onar, Başar Öztayşi \\ Istanbul Technical University, Department of Industrial Engineering, 34367 Macka, Besiktas, \\ Istanbul, Turkey \\ *kahramanc@itu.edu.t
}

\begin{abstract}
Hesitant fuzzy sets are an extension of ordinary fuzzy sets. They are composed of dual hesitant fuzzy sets, interval valued hesitant fuzzy sets, generalized hesitant fuzzy sets, hesitant fuzzy linguistic term sets, and triangular fuzzy hesitant fuzzy sets. Multiexperts evaluations are integrated by aggregation operators. This paper develops the fuzzy present worth analysis using triangular hesitant fuzzy sets and aggregation operators and includes a numerical example.
\end{abstract}

Keywords: Present worth, uncertainty, hesitant, fuzzy sets, triangular fuzzy number, economic analysis

\section{Introduction}

A future amount of money converted to its equivalent present value is called a present worth (PW). Present worth values are often referred to as discounted cash flows (DCF). Besides PW, equivalent terms frequently used are present value (PV) and net present worth (NPW). The present worth analysis (PWA) is one of the most popular three techniques in capital budgeting. They are PWA, Annual Cash Flow Analysis (ACFA), and Rate of Return Analysis (RORA). In present worth analysis, the $\mathrm{P}$ value is calculated at the minimum attractive rate of return (MARR) for each alternative. This converts all future cash flows into present dollar equivalents. This makes it easy to determine the economic advantage of one alternative over another [1],[2]

The values of parameters used in PWA such as MARR, life, and cash flows are generally determined under risk or uncertainty conditions rather than deterministic conditions. If sufficient observations exist for a certain parameter, risk analysis can be applied since the probability distribution of that parameter can be obtained. If sufficient observations do not exist for a certain parameter, the analyses under uncertainty are applied. Some decision criteria such as Laplace, Hurwicz, and minimum regret, which are still based on probabilistic calculations, can be used under uncertainty conditions.

The fuzzy set theory is an excellent tool to handle the uncertainty in case of insufficient data. Its principals are similar to human beings' thinking style. Ordinary fuzzy sets have recently been extended to hesitant fuzzy sets, intuitionistic fuzzy sets, type-2 fuzzy sets, nonstationary fuzzy sets, and fuzzy multisets [3].
Hesitant Fuzzy Sets (HFS) were introduced by Torra [4] as a new extension of fuzzy sets, motivated for the common difficulty that often appears when the membership degree of an element must be established and the difficulty is not because of an error margin (as in IFS) or due to some possibility distribution (as in T2FS), but rather because there are some possible values that make to hesitate about which one would be the right one. This situation is very usual in decision making when an expert might consider different degrees of membership of an element $x$ in the set $\mathrm{A}$ [3].

In this paper, to the best of our knowledge, the present worth analysis is first time realized by using hesitant fuzzy sets. Discount rate and cash flows are accepted to be hesitant fuzzy sets. Numerical examples are given for equal and different lives alternatives and infinite life alternatives.

The rest of the paper is organized as follows. Section 2 summarizes the present worth analysis. Section 3 gives the basics and arithmetic operations of hesitant fuzzy sets. Section 4 includes numerical examples for hesitant fuzzy present worth analysis. Section 5 concludes the paper and includes suggestions for further research.

\section{Present Worth Analysis}

The PW comparison of alternatives with equal lives is straightforward. If both alternatives are used in identical capacities for the same time period, they are termed equal-service alternatives. If only one alternative exists, its $\mathrm{PW}$ is calculated at the MARR and if $\mathrm{PW} \geq 0$, the alternative is financially viable. For two or more mutually exclusive alternatives, the NPW of each alternative is calculated at the MARR and the alternative with the NPW value that is numerically largest, that is, less negative or more positive is selected. If it is a fixed input problem, the alternative with maximum present worth of benefits (PWB) is selected. If it is a fixed output problem, the alternative with minimum present worth of costs (PWC) is selected.

\section{Hesitant Fuzzy Sets}

HFS is a novel and recent extension of fuzzy sets that aims to model the uncertainty originated by the hesitation that might arise in the assignment of membership degrees of the elements to a fuzzy set. A HFS is defined in terms of a function that returns a set of membership values for each element in the domain. 
Definition 1. Let $X$ be a reference set, a HFS on $X$ is a function $\mathrm{f}$ that returns a subset of values in $[0,1]$ (Torra, 2010):

$$
\text { f }: X \rightarrow \varphi([0,1])
$$

Definition 2. Let $M=\left\{\mu_{1}, \mu_{2}, \ldots, \mu_{n}\right\}$ be a set of $n$ membership functions. The HFS associated to $\mathrm{M}, \mathrm{f}_{M}$, is defined as [4]:

$$
\begin{gathered}
\mathfrak{\wp}_{M}: X \rightarrow \varphi([0,1]) \\
\oiiint_{M}(x)=\cup_{\mu \in M}\{\mu(x)\}
\end{gathered}
$$

where $x \in X$.

$\mathrm{Xia}$ and $\mathrm{Xu}$ [5] completed the original definition of HFS by including the mathematical representation of a HFS as follows:

$$
E=\left\{\left\langle x, h_{E}(x)\right\rangle: x \in X\right\}
$$

where $h_{E}(x)$ is a set of some values in [0,1], denoting the possible membership degrees of the element $x \in X$ to the set $E$. For convenience, $\mathrm{Xia}$ and $\mathrm{Xu}$ [5] noted $\mathrm{h}=$ $h_{E}(x)$ and called it Hesitant Fuzzy Element (HFE) of $E$ and $H=\cup h_{E}(x)$, the set of all HFEs of $E$.

The concepts, basic operations and properties defined for HFSs and HFEs cope with the hesitation of assigning a membership degree of an element to a fuzzy set. The idea of modelling such a hesitation has been extended together with the previous methods and tools to the following three situations: (i) to model the hesitation not only for the assignment of the membership degree, but also for the non-membership degree; (ii) to manage the hesitation on membership degrees that are not exactly defined, but expressed by interval values, intuitionistic fuzzy sets, or triangular fuzzy numbers; (iii) to deal with the hesitation in qualitative settings in which information is linguistically modelled [6].

The extensions of hesitant fuzzy sets can be classified as follows:

\section{Dual hesitant fuzzy sets}

Zhu et al.[7] proposed the concept of Dual Hesitant Fuzzy Set (DHFS), as an extension of HFS to deal with the hesitation both for the membership degree and nonmembership degree. A DHFS is defined in terms of two functions that return two sets of membership and nonmembership values respectively for each element in the domain as follows:

Definition 3. Let $X$ be a set, a DHFS D on $X$ is defined as:

$$
D=\{<x, h(x), g(x)>\mid x \in X\}
$$

where $h(x)$ and $g(x)$ are two sets of values in the interval $[0,1]$, denoting the possible membership and non- membership degrees of the element $x \in X$ to the set $D$, respectively.

\section{Interval valued hesitant fuzzy sets}

Chen et al. [8] presented the definition of IntervalValued Hesitant Fuzzy Set (IVHFS), as a generalization of HFS in which the membership degrees of an element to a given set are defined by several possible interval values. An IVHFS is defined as follows:

Definiton 4. Let $X$ be a reference set, and $I([0,1])$ be a set of all closed subintervals of $[0,1]$. An IVHFS on $X$ is,

$$
\tilde{A}=\left\{\left\langle x_{i}, \tilde{h}_{A}\left(x_{i}\right)\right\rangle \mid x_{i} \in X, i=1,2, \ldots, n\right\}
$$

where $\tilde{h}_{A}\left(x_{i}\right): \mathrm{X} \rightarrow \varphi(\mathrm{I}([0,1]))$ denotes all possible interval-valued membership degrees of the element $x_{i} \in$ $X$ to the set $\tilde{A}$.

\section{Generalized hesitant fuzzy sets}

Qian et al. [9] extended the concept of HFS by Atanassov's intuitionistic fuzy sets (IFS). The idea consists of representing the membership as the union of some Atanassov's IFS. In order to define this new extension called Generalized Hesitant Fuzzy Set (GHFS), authors use Eq. (2) introduced by Torra.

Definition 5. Given a set of $\mathrm{n}$ membership functions:

$$
\mathrm{M}=\left\{\alpha_{\mathrm{i}}=\mu_{\mathrm{i}} \mathrm{v}_{\mathrm{i}} \mid 0 \leq \mu_{\mathrm{i}}, \mathrm{v}_{\mathrm{i}} \leq 1,0 \leq \mu_{\mathrm{i}}+\mathrm{v}_{\mathrm{i}} \leq 1, \mathrm{i}=\right.
$$

the GHFS associated to $\mathrm{M}, \mathrm{f}_{M}$, is defined as follows:

$$
\mathfrak{f}_{M}(x)=\mathrm{U}_{\left(\mu_{i}(x), v_{i}(x)\right) \epsilon M}\left(\mu_{i}(x), v_{i}(x)\right)
$$

\section{Hesitant fuzzy linguistic term sets}

Rodriguez et al. [3] proposed the concept of Hesitant Fuzzy Linguistic Term Set (HFLTS) which keeps the basis on the fuzzy linguistic approach and extends the idea of HFS to linguistic contexts.

Definition 6. Let $S=\left\{s_{0}, s_{1}, \ldots s_{g}\right\}$ be a linguistic term set, a HFLTS, $H_{S}$, is defined as an ordered finite subset of consecutive linguistic terms of $\mathrm{S}$ :

$$
H_{S}=\left\{s_{i}, s_{i+1}, \ldots, s_{j}\right\} \text { such that } s_{k} \in S, k \in\{i, \ldots, j\}
$$

\section{Triangular fuzzy hesitant fuzzy sets}

$\mathrm{Yu}$ [10] introduced the concept of Triangular Fuzzy Hesitant Fuzzy Set (TFHFS), whose membership degrees of an element to a fuzzy set are expressed by several triangular fuzzy numbers.

Definition 7. Let $X$ be a fixed set, a TFHFS $\tilde{E}$ on $X$ is defined in terms of a function $\tilde{f}_{\tilde{E}}(x)$ that returns several triangular fuzzy values,

$$
\tilde{E}=\left\{<x, \tilde{f}_{\tilde{E}}(x)>\mid x \in X\right\}
$$


where $\tilde{f}_{\tilde{E}}(x)$ is a set of several triangular fuzzy numbers which express the possible membership degrees of an element $x \in X$ to a set $\tilde{E}$.

Definition 8. For a Triangular Fuzzy Hesitant Fuzzy Set (TFHFS), $\tilde{f}, s(\tilde{f})=\frac{1}{l_{\tilde{f}}} \sum_{\overparen{T F N} \in \tilde{f}} \bar{X}(\widetilde{T F N})$ is called the score function of $\tilde{f}$ with $l_{\tilde{f}}$ being the number of TFNs in $\tilde{f}$ [10]. $h(\tilde{f})=\frac{1}{l_{\tilde{f}}} \sum_{\overparen{T F N} \in \tilde{f}} \sigma(\widetilde{T F N})$ is called the deviation function of $\tilde{f}$. For $\tilde{f}_{1}$ and $\tilde{f}_{2}$,

i. If $s\left(\tilde{f}_{1}\right)>s\left(\tilde{f}_{2}\right)$, then $\tilde{f}_{1} \geq \tilde{f}_{2}$,

ii. If $s\left(\tilde{f}_{1}\right)=s\left(\tilde{f}_{2}\right), h\left(\tilde{f}_{1}\right)=h\left(\tilde{f}_{2}\right)$, then $\tilde{f}_{1}=\tilde{f}_{2}$

iii. If $s\left(\tilde{f}_{1}\right)=s\left(\tilde{f}_{2}\right), h\left(\tilde{f}_{1}\right)>h\left(\tilde{f}_{2}\right)$, then $\tilde{f}_{1}<\tilde{f}_{2}$

iv. If $s\left(\tilde{f}_{1}\right)=s\left(\tilde{f}_{2}\right), h\left(\tilde{f}_{1}\right)<h\left(\tilde{f}_{2}\right)$, then $\tilde{f}_{1}>\tilde{f}_{2}$

Definition 9. Let $\tilde{f}_{1}$ and $\tilde{f}_{2}$ be two THHFEs, then

$\tilde{f}_{1} \oplus \tilde{f}_{2}=\left\{\left(L_{1}+L_{2}-L_{1} \cdot L_{2}, M_{1}+M_{2}-\right.\right.$

$\left.\left.M_{1} \cdot M_{2}, U_{1}+U_{2}-U_{1} \cdot U_{2}\right) \mid \overparen{T F N}_{1} \in \tilde{f}_{1}, \overparen{T F N}_{2} \in \tilde{f}_{2}\right\}$

$\tilde{f}_{1} \otimes \tilde{f}_{2}=\left\{L_{1} \cdot L_{2}, M_{1} \cdot M_{2}, U_{1} \cdot U_{2} \mid \widetilde{T F N}_{1} \in \tilde{f}_{1}, \widetilde{T F N}_{2} \in\right.$ $\left.\tilde{f}_{2}\right\}$

$\tilde{f}^{\lambda}=\left\{(L)^{\lambda},(M)^{\lambda},(U)^{\lambda} \mid \widetilde{T F N} \in \tilde{f}\right\}, \lambda>0$

$\lambda \tilde{f}=\left\{1-(1-L)^{\lambda}, 1-(1-M)^{\lambda}, 1-(1-\right.$

$\left.U)^{\lambda} \mid \widetilde{T F N} \in \tilde{f}\right\}, \lambda>0$

where $\widetilde{T F N}_{1}=\left(L_{1}, M_{1}, U_{1}\right)$ and $\widetilde{T F N}_{2}=\left(L_{2}, M_{2}, U_{2}\right)$

\section{Aggregation Operators for Triangular Fuzzy Hesitant Fuzzy Sets}

The aggregation problem consists of aggregating ntuples of objects all belonging to a given set, into a single object of the same set. In other words, aggregation operations on fuzzy numbers are operations by which several fuzzy numbers are combined to produce a single fuzzy number.

Definition 10. Let $\tilde{f}_{j}(j=1,2, \ldots, n)$ be a collection of TFHFEs. $w=\left(w_{1}, w_{2}, \ldots, w_{n}\right)^{T}$ is the weight vector of $\tilde{f}_{j}(j=1,2, \ldots, n)$ with $w_{j} \in[0,1]$ and $\sum_{j=1}^{n} w_{j}=1$, then a Triangular Fuzzy Hesitant Fuzzy Weighted Averaging (TFHFWA) operator is a mapping TFHFWA: $F^{n} \rightarrow \bar{F}$ such that

$$
\begin{aligned}
& \operatorname{TFHFWA}\left(\tilde{f}_{1}, \tilde{f}_{2}, \ldots, \tilde{f}_{n}\right)=\bigoplus_{j=1}^{n}\left(w_{i} \tilde{f}_{j}\right)=\{1- \\
& \prod_{j=1}^{n}\left(1-L_{j}\right)^{w_{j}}, 1-\prod_{j=1}^{n}\left(1-M_{j}\right)^{w_{j}}, 1-\prod_{j=1}^{n}(1-
\end{aligned}
$$

$\left.\left.U_{j}\right)^{w_{j}} \mid \widetilde{T F N}_{1} \in \tilde{f}_{1}, \widetilde{T F N}_{1} \in \tilde{f}_{1}, \ldots, \widetilde{T F N}_{n} \in \tilde{f}_{n}\right\}$

Definition 11. Alternatively, a Triangular Fuzzy Hesitant Fuzzy Weighted Geometric (TFHFWG) operator is a mapping IVHFWG: $F^{n} \rightarrow \bar{F}$ such that

$\operatorname{TFHFWG}\left(\tilde{f}_{1}, \tilde{f}_{2}, \ldots, \tilde{f}_{n}\right)=\bigotimes_{j=1}^{n} \tilde{f}_{j}{ }^{w_{j}}=$ $\left\{\left(\prod_{j=1}^{n}\left(L_{j}\right)^{w_{j}}, \prod_{j=1}^{n}\left(M_{j}\right)^{w_{j}}, \prod_{j=1}^{n}\left(U_{j}\right)^{w_{j}}\right) \mid \widetilde{T F N}_{1} \in\right.$ $\left.\tilde{f}_{1}, \widetilde{T F N}_{1} \in \tilde{f}_{1}, \ldots, \widetilde{T F N}_{n} \in \tilde{f}_{n}\right\}$

\section{Hesitant Fuzzy Present Worth Analysis}

In the hesitant fuzzy present worth analysis, triangular fuzzy hesitant fuzzy sets are used to express the investment parameters. The handled parameters are first cost (FC), uniform annual cost (UAC), uniform annual benefit (UAB), project life $(n)$, interest rate (i), and salvage value $(\mathrm{SV})$. These parameters are expressed by triangular fuzzy hesitant fuzzy sets as follows:

$$
\begin{aligned}
& \widetilde{F C}=\left\{\begin{array}{c}
\left\langle f c_{1}, T F N_{1}, \ldots, T F N_{m}\right\rangle,\left\langle f c_{2}, T F N_{1}, \ldots, T F N_{m}\right\rangle, \\
\ldots,\left\langle f c_{k}, T F N_{1}, \ldots, T F N_{m}\right\rangle
\end{array}\right\} \\
& \widetilde{U A C}=\left\{\begin{array}{c}
\left\langle u a c_{1}, T F N_{1}, \ldots, T F N_{m}\right\rangle, \\
\left\langle u a c_{2}, T F N_{1}, \ldots, T F N_{m}\right\rangle, \\
\ldots,\left\langle u a c_{k}, T F N_{1}, \ldots, T F N_{m}\right\rangle
\end{array}\right\} \\
& \widetilde{U A B}=\left\{\begin{array}{c}
\left\langle u a b_{1}, T F N_{1}, \ldots, T F N_{m}\right\rangle, \\
\left\langle u, T u a b_{k}, T F N_{1}, \ldots, T F N_{m}\right\rangle, \\
\left.\ldots F N_{m}\right\rangle
\end{array}\right\} \\
& \widetilde{S V}=\left\{\begin{array}{c}
\left\langle s v_{1}, T F N_{1}, \ldots, T F N_{m}\right\rangle, \\
\left\langle s v_{2}, T F N_{1}, \ldots, T F N_{m}\right\rangle, \\
\ldots,\left\langle s v_{k}, T F N_{1}, \ldots, T F N_{m}\right\rangle
\end{array}\right\} \\
& \tilde{\imath}=\left\{\begin{array}{c}
\left\langle i_{1}, T F N_{1}, \ldots, T F N_{m}\right\rangle, \\
\left\langle i_{2}, T F N_{1}, \ldots, T F N_{m}\right\rangle, \\
\ldots,\left\langle i_{k}, T F N_{1}, \ldots, T F N_{m}\right\rangle
\end{array}\right\} \\
& \tilde{n}=\left\{\begin{array}{c}
\left\langle n_{1}, T F N_{1}, \ldots, T F N_{m}\right\rangle, \\
\left\langle n_{2}, T F N_{1}, \ldots, T F N_{m}\right\rangle, \\
\ldots,\left\langle n_{k}, T F N_{1}, \ldots, T F N_{m}\right\rangle
\end{array}\right\}
\end{aligned}
$$

Using the hesitant fuzzy parameters above, the present worth of an investment alternative can be calculated using the following equations.

$$
\begin{aligned}
& \widetilde{P W}=-\widetilde{F C}-\widetilde{U A C}\left(\frac{P}{A}, \tilde{\imath}, \tilde{n}\right)+\widetilde{U A B}\left(\frac{P}{A}, \tilde{l}, \tilde{n}\right)+ \\
& \widetilde{S V}\left(\frac{P}{F}, \tilde{\imath}, \tilde{n}\right)
\end{aligned}
$$

$\widetilde{P W}=-\widetilde{F C}-\widetilde{U A C}\left[\frac{(1+\tilde{l})^{\widetilde{n}}-1}{\tilde{i}(1+\tilde{\imath})^{\tilde{n}}}\right]+\widetilde{U A B}\left[\frac{(1+\tilde{i})^{\tilde{n}}-1}{\tilde{\imath}(1+\tilde{\imath})^{\tilde{n}}}\right]+$ $\widetilde{S V}(1+\tilde{l})^{-\tilde{n}}$ 
Aggregation of triangular hesitant fuzzy sets is performed by Eq. 14 or Eq. 15. Later, average parameter values can be calculated by multiplying the defuzzified values of membership functions with possible parameter values. The defuzzification of parameters at the latest stage easifies the calculation of present worth.

\section{Application}

The parameters of an investment alternative have the possible values and triangular hesitant fuzzy membership values given in Table 1. Realizing the aggregation, defuzzification, and averaging operations, a single value for each parameter is obtained. Tables 2 and 3 show the results of these operators. With TFHFWA aggregation:

$\mathrm{PW}=-16,073.77+(8,033.556-3,000.961) *(\mathrm{P} / \mathrm{A}, 6.81 \%$, $12.321)+4,991.636 *(\mathrm{P} / \mathrm{F}, 6.81 \%, 12.321)=\$ 27,224.64$ With TFHFWG aggregation:

$\mathrm{PW}=16,063.63+(8,031.927-2,987.835) *(\mathrm{P} / \mathrm{A}, 6.8 \%$, $12.32)+4,989.738 *(\mathrm{P} / \mathrm{F}, 6.8 \%, 12.32)=\$ 27,350.85$

\begin{tabular}{|l|c|c|c|c|}
\hline \multirow{2}{*}{} & \multirow{3}{*}{} & \multicolumn{3}{|c|}{ Experts' weights } \\
\cline { 3 - 5 } & & & & \\
\cline { 3 - 5 } Parameter & Pos. va. & 0.5 & 0.3 & 0.2 \\
\hline FC & $\$ 15,000$ & $(0.4,0.5,0.7)$ & $(0.3,0.4,0.6)$ & $(0.4,0.5,0.6)$ \\
\hline & $\$ 16,000$ & $(0.5,0.7,0.8)$ & $(0.3,0.5,0.6)$ & $(0.4,0.5,0.6)$ \\
\hline & $\$ 17,000$ & $(0.5,0.7,0.9)$ & $(0.3,0.4,0.7)$ & $(0.4,0.6,0.8)$ \\
\hline UAC & $\$ 2,000$ & $(0.1,0.3,0.4)$ & $(0.2,0.4,0.5)$ & $(0.3,0.5,0.6)$ \\
\hline & $\$ 3,000$ & $(0.1,0.2,0.4)$ & $(0.3,0.4,0.6)$ & $(0.3,0.4,0.6)$ \\
\hline & $\$ 4,000$ & $(0.1,0.3,0.3)$ & $(0.3,0.4,0.6)$ & $(0.3,0.5,0.5)$ \\
\hline UAB & $\$ 7,000$ & $(0.6,0.7,0.7)$ & $(0.5,0.6,0.7)$ & $(0.5,0.5,0.6)$ \\
\hline & $\$ 8,000$ & $(0.6,0.7,0.8)$ & $(0.6,0.7,0.8)$ & $(0.4,0.5,0.6)$ \\
\hline & $\$ 9,000$ & $(0.5,0.7,0.8)$ & $(0.4,0.7,0.8)$ & $(0.7,0.8,0.9)$ \\
\hline SV & $\$ 4,000$ & $(0.3,0.4,0.4)$ & $(0.3,0.4,0.6)$ & $(0.4,0.5,0.6)$ \\
\hline & $\$ 5,000$ & $(0.5,0.6,0.7)$ & $(0.4,0.6,0.8)$ & $(0.3,0.6,0.7)$ \\
\hline & $\$ 6,000$ & $(0.3,0.4,0.5)$ & $(0.3,0.4,0.7)$ & $(0.2,0.3,0.4)$ \\
\hline İ & $6 \%$ & $(0.7,0.8,0.9)$ & $(0.8,0.8,0.9)$ & $(0.6,0.8,0.8)$ \\
\hline & $7 \%$ & $(0.5,0.7,0.7)$ & $(0.5,0.6,0.6)$ & $(0.4,0.5,0.7)$ \\
\hline & $8 \%$ & $(0.3,0.4,0.5)$ & $(0.3,0.4,0.7)$ & $(0.4,0.5,0.6)$ \\
\hline N & 10 years & $(0.4,0.5,0.7)$ & $(0.3,0.5,0.6)$ & $(0.4,0.5,0.6)$ \\
\hline & 12 years & $(0.5,0.7,0.8)$ & $(0.5,0.6,0.8)$ & $(0.5,0.7,0.7)$ \\
\hline & 14 years & $(0.8,0.8,0.9)$ & $(0.7,0.8,0.9)$ & $(0.7,0.9,0.9)$ \\
\hline
\end{tabular}

Table 1. Possible values and triangular hesitant fuzzy membership values

The evaluations of different experts are aggregated to obtain a single aggregated value. Table 2 represents the aggregated value of each parameter. As an example, the value $(0.372,0.472,0.654)$ in Table 2 is calculated by TFHFWA using Eq.14 as follows:

$$
\begin{gathered}
\begin{aligned}
1-\left[(1-0.4)^{0.5}\right. & \left.(1-0.3)^{0.3}(1-0.4)^{0.2}\right] \\
& =0.372 \\
1-\left[(1-0.5)^{0.5}\right. & \left.(1-0.4)^{0.3}(1-0.5)^{0.2}\right] \\
& =0.472 \\
1-\left[(1-0.7)^{0.5}\right. & \left.(1-0.6)^{0.3}(1-0.6)^{0.2}\right] \\
& =0.654
\end{aligned}
\end{gathered}
$$

The next step is to determine the defuzzified value of each parameter. Table 7 presents the defuzzified values of the parameters. As an example of the calculations, the value $16,146.160$ in Table 7 is determined as follows:

First of all, defuzzified values of all possible values are calculated using Eq 24.

\begin{tabular}{|c|c|c|c|c|}
\hline & Pos. valu. & TFHFWA aggregation & Def. & Avrg \\
\hline \multirow[t]{3}{*}{$\mathrm{FC}$} & $\$ 15,000$ & $(0.372,0.472,0.654)$ & 0.493 & 16,073 \\
\hline & $\$ 16,000$ & $(0.426,0.613,0.717)$ & 0.592 & \\
\hline & $\$ 17,000$ & $(0.426,0.609,0.84)$ & 0.621 & \\
\hline \multirow[t]{3}{*}{$\mathrm{UAC}$} & $\$ 2,000$ & $(0.174,0.375,0.476)$ & 0.350 & 3,000 \\
\hline & $\$ 3,000$ & $(0.206,0.307,0.51)$ & 0.333 & \\
\hline & $\$ 4,000$ & $(0.206,0.375,0.447)$ & 0.351 & \\
\hline \multirow[t]{3}{*}{$\mathrm{UAB}$} & $\$ 7,000$ & $(0.553,0.638,0.682)$ & 0.628 & 8,033 \\
\hline & $\$ 8,000$ & $(0.566,0.668,0.77)$ & 0.668 & \\
\hline & $\$ 9,000$ & $(0.523,0.723,0.826)$ & 0.699 & \\
\hline \multirow[t]{3}{*}{ SV } & $\$ 4,000$ & $(0.321,0.421,0.51)$ & 0.418 & 4,991 \\
\hline & $\$ 5,000$ & $(0.435,0.6,0.734)$ & 0.592 & \\
\hline & $\$ 6,000$ & $(0.281,0.381,0.555)$ & 0.400 & \\
\hline \multirow[t]{3}{*}{$\mathrm{i}$} & $6 \%$ & $(0.719,0.8,0.885)$ & 0.801 & 0.068 \\
\hline & $7 \%$ & $(0,481,0,638,0,673)$ & 0.608 & \\
\hline & $8 \%$ & $(0,321,0,421,0,590)$ & 0.438 & \\
\hline \multirow[t]{3}{*}{$\mathrm{n}$} & 10 years & $(0,372,0,500,0,654)$ & 0.507 & 12.321 \\
\hline & 12 years & $(0,500,0,673,0,783)$ & 0.657 & \\
\hline & 14 years & $(0,755,0,826,0,900)$ & 0.827 & \\
\hline
\end{tabular}

$\operatorname{Def}(\widetilde{T F N})=\frac{l+2 m+u}{4}$

where $\widetilde{\boldsymbol{T F N}}=(\boldsymbol{l}, \boldsymbol{m}, \boldsymbol{u})$

$$
\begin{aligned}
& \frac{0.372+2 * 0.472+0.654}{4}=0.492 \\
& \frac{0.426+2 * 0.613+0.717}{4}=0.592 \\
& \frac{0.426+2 * 0.609+0.84}{4}=0.621
\end{aligned}
$$

Table 2. TFHFWA aggregation \&defuzzification of investment parameters

In the second step of the study, the evaluations of different experts are aggregated using TFHFW. Table 3 represents the aggregated value of each parameter. As an example of the calculations the value $(0.367,0.468$, 0.648) in Table 3 is calculated using Eq. 15 as follows:

$$
\left[(0.4)^{0.5} \times(0.3)^{0.3} \times(0.4)^{0.2}\right]=0.367
$$




$$
\begin{aligned}
& {\left[(0.5)^{0.5} \times(0.4)^{0.3} \times(0.5)^{0.2}\right]=0.468} \\
& {\left[(0.7)^{0.5} \times(0.6)^{0.3} \times(0.6)^{0.2}\right]=0.648}
\end{aligned}
$$

These values are later defuzzified using Eq. 24.

\begin{tabular}{|c|c|c|c|c|}
\hline & Pos. valu. & TFHFWG aggregation & Def. & Avrg \\
\hline \multirow[t]{3}{*}{$\mathrm{FC}$} & $\$ 15,000$ & $(0.367,0.468,0.648)$ & 0.488 & 16,063 \\
\hline & $\$ 16,000$ & $(0.41,0.592,0.693)$ & 0.572 & \\
\hline & $\$ 17,000$ & $(0.41,0.574,0.815)$ & 0.593 & \\
\hline \multirow[t]{3}{*}{ UAC } & $\$ 2,000$ & $(0.153,0.362,0.464)$ & 0.335 & 2,987 \\
\hline & $\$ 3,000$ & $(0.173,0.283,0.49)$ & 0.307 & \\
\hline & $\$ 4,000$ & $(0.173,0.362,0.409)$ & 0.327 & \\
\hline \multirow[t]{3}{*}{$\mathrm{UAB}$} & $\$ 7,000$ & $(0.548,0.625,0.679)$ & 0.619 & 8,031 \\
\hline & $\$ 8,000$ & $(0.553,0.654,0.755)$ & 0.654 & \\
\hline & $\$ 9,000$ & $(0.5,0.719,0.819)$ & 0.689 & \\
\hline \multirow[t]{3}{*}{ SV } & $\$ 4,000$ & $(0.318,0.418,0.49)$ & 0.411 & 4,989 \\
\hline & $\$ 5,000$ & $(0.422,0.6,0.729)$ & 0.588 & \\
\hline & $\$ 6,000$ & $(0.277,0.378,0.529)$ & 0.391 & \\
\hline \multirow[t]{3}{*}{$\mathrm{i}$} & $6 \%$ & $(0.706,0.8,0.879)$ & 0.796 & 0.068 \\
\hline & $7 \%$ & $(0.478,0.625,0.668)$ & 0.599 & \\
\hline & $8 \%$ & $(0.318,0.418,0.574)$ & 0.432 & \\
\hline \multirow[t]{3}{*}{$\mathrm{n}$} & 10 years & $(0.367,0.5,0.648)$ & 0.504 & 12.32 \\
\hline & 12 years & $(0.5,0.668,0.779)$ & 0.654 & \\
\hline & 14 years & $(0.748,0.819,0.9)$ & 0.822 & \\
\hline
\end{tabular}

$$
\frac{0.367+2 * 0.468+0.648}{4}=0.488
$$

Table 3. TFHFWA aggregation \&defuzzification of investment parameters

\section{References}

1. L., B. and T. A., Engineering Economy. 2012, New York: McGraw-Hill.

2. Kahraman, C., Fuzzy Engineering Economics with Applications. 2008, Berlin: Springer.

3. Rodriguez, R.M., L. Martinez, and F. Herrera, Hesitant Fuzzy Linguistic Term Sets for Decision Making. Fuzzy Systems, IEEE Transactions on, 2012. 20(1): p. 109-119.

4. Torra, V., Hesitant fuzzy sets. International Journal of Intelligent Systems, 2010. 25(6): p. 529-539.

5. Xia, M.M. and Z.S. Xu, Hesitant fuzzy information aggregation in decision making. International Journal Approximate Reasoning, 2011. 52: p. 395-40.

6. Rodríguez, R.M., et al., Hesitant Fuzzy Sets: State of the Art and Future Directions. International Journal of Intelligent Systems, 2014. 29(6): p. 495-524.

\section{Conclusion and further suggestions}

Hesitant fuzzy sets have been extensively used in the literature since they effectively incorporate the different experts ideas on a single set. The proposed present worth algorithm combines the different experts membership functions by using an aggregation operator and then defuzzifies the obtained single membership function. The defuzzified value and the possible discrete values of parameters are used to calculate the average parameter value in a similar way of expected value concept. Later, the average values are used in the classical present worth analysis. We suggest other extensions of hesitant fuzzy sets to be used for the same aim for further research.

7. Zhu, B., Z.S. Xu, and M.M. Xia, Dual hesitant fuzzy sets. Journal of Applied Mathematics, 2012: p. 1-13.

8. Chen, N., Z.S. Xu, and M.M. Xia, Intervalvalued hesitant preference relations and their applications to group decision making. Knowledge-Based Systems, 2013. 37(2): p. 528-540.

9. Qian, G., H. Wang, and X. Feng, Generalized hesitant fuzzy sets and their application in decision support system. Knowledge-Based Systems, 2013. 37(1): p. 357-365.

10. Yu, D. Triangular Hesitant Fuzzy Set and Its Application to Teaching Quality Evaluation. Journal of Information \& Computational Science, 2013. 10(7): p. 1925-1934. 